\title{
Inhibitor of prostacyclin production in sporadic haemolytic uraemic syndrome
}

\author{
M LEVIN, K B ELKON, T J C NOKES, A M BUCKLE, M J DILLON, \\ R M HARDISTY, AND T M BARRATT
}

Departments of Nephrology and Haematology, Institute of Child Health, London

SUMmaRY Prostacyclin ( $\left.\mathrm{PGI}_{2}\right)$ production was diminished when rat aortic rings were incubated with plasma from 5 of 6 patients with the sporadic form of haemolytic uraemic syndrome but was normal in the presence of plasma from 7 patients with the epidemic form of haemolytic uraemic syndrome or from patients with other renal diseases. The reduced $\mathrm{PGI}_{2}$ production was caused by an unstable inhibitor, extractable into polar lipid solvents, in sporadic haemolytic uraemic plasma. These results suggest that there may be at least 2 different pathogenetic mechanisms in epidemic and sporadic haemolytic uraemic syndrome and that the reduced $\mathrm{PGI}_{2}$ production observed in the sporadic type is due to an inhibitor of $\mathrm{PGI}_{2}$ production rather than a deficiency of stimulating factors.

Prostacyclin $\left(\mathrm{PGI}_{2}\right)$ deficiency has been implicated in the pathogenesis of the haemolytic uraemic syndrome (HUS) and thrombotic thrombocytopenic purpura (TTP), ${ }^{1-4}$ but there are conflicting views on the pathogenetic mechanisms. Plasma from some patients with HUS shows a reduced capacity to support $\mathrm{PGI}_{2}$ production by endothelial tissue and this has been ascribed to a deficiency of a factor present in normal plasma ${ }^{5}$ which stimulates PGI production. ${ }^{1}$ This hypothesis is supported by the observation that fresh plasma infusions induce remission in some patients with HUS and TTP. ${ }^{16} 7$ Other patients, however, have responded instead to plasma exchange, ${ }^{7}$ suggesting that removal of an inhibitor of $\mathrm{PGI}_{2}$ production may be implicated. More recently excessively rapid degradation of $\mathrm{PGI}_{2}$ in HUS plasma has been observed and has been proposed as a third possible explanation for the $\mathrm{PGI}_{2}$ deficiency. ${ }^{4}$ The interpretation of the importance of $\mathrm{PGI}_{2}$ deficiency in HUS is further complicated by the fact that it is not a constant feature of the syndrome and that trials of $\mathrm{PGI}_{2}$ infusions have had only moderate success in reversing the condition. ${ }^{8}$

Although the heterogeneity of patients with HUS and TTP has been recognised, ${ }^{9}{ }^{10}$ most reports on $\mathrm{PGI}_{2}$ production have failed to distinguish the different subgroups within the HUS spectrum. Epidemiological studies on HUS in children, however, suggest the existence of at least 2 distinct subgroups in the United Kingdom. ${ }^{11}$ An epidemic form occurs mainly in summer, in younger children, usually with an explosive onset after diarrhoea, and has a good prognosis. In contrast a sporadic form occurs in older children, with no seasonal variation, developing insidiously without clear prodromal illness or after an upper respiratory infection, and the prognosis for renal function is poor.

In addition to the childhood epidemic and sporadic forms, HUS is seen in association with pregnancy, ${ }^{12}$ with collagen vascular disease, ${ }^{12}$ after some infections, ${ }^{12} 13$ and with drug exposure. ${ }^{12}$ Clearly, different pathogenetic mechanisms may underlie these different forms of HUS and may account for the disparate results obtained in studies on pathogenesis and in treatment trials in these disorders.

We show that plasma of patients with sporadic HUS (but not epidemic HUS) fails to support PGI $_{2}$ production by rat aortic rings and that this abnormality is due to the presence of an inhibitory substance. The results of preliminary studies on the nature of the inhibitor are reported.

\section{Patients}

HUS was diagnosed in 13 children with microangiopathic haemolytic anaemia, renal faliure, and thrombocytopenia in whom other causes, particularly septicaemia, had been excluded (Table). Seven younger children aged 11 months to 7 years (cases 1-7) in whom HUS developed acutely after a diarrhoeal prodrome during a summer epidemic were classified as having epidemic HUS. Five older 703 
Table Clinical details of patients with haemolytic uraemic syndrome (HUS) and the effect of their plasma on PGI production by aortic rings

\begin{tabular}{|c|c|c|c|c|c|c|c|}
\hline Case No & Age (years) & $\begin{array}{l}\text { Prodromal } \\
\text { diarrhoea }\end{array}$ & $\begin{array}{l}\text { Season of } \\
\text { onset }\end{array}$ & $\begin{array}{l}\text { Highest plasma } \\
\text { creatinine } \\
(\mu \mathrm{mol} / \mathrm{l})\end{array}$ & $\begin{array}{l}\text { Lowest platelet } \\
\text { count } \times 10^{9} / l\end{array}$ & Type of HUS & $\begin{array}{l}\mathrm{PGI}_{2} \\
\text { production }\end{array}$ \\
\hline 1 & 0.9 & Yes & Summer & 550 & 45 & Epidemic HUS & Normal \\
\hline 2 & $1 \cdot 2$ & Yes & Summer & 443 & 63 & Epidemic HUS & Normal \\
\hline 3 & $2 \cdot 0$ & Yes & Summer & 600 & 40 & Epidemic HUS & Normal \\
\hline 4 & $2 \cdot 1$ & Yes & Summer & 580 & 45 & Epidemic HUS & Normal \\
\hline 5 & $5 \cdot 5$ & Yes & Summer & 1000 & 70 & Epidemic HUS & Normal \\
\hline 6 & $5 \cdot 5$ & Yes & Summer & 260 & 80 & Epidemic HUS & Normal \\
\hline 7 & $7 \cdot 0$ & Yes & Summer & 1300 & 90 & Epidemic HUS & Normal \\
\hline 8 & $5 \cdot 0$ & No & Winter & 460 & 60 & Sporadic HUS & Reduced \\
\hline 9 & $7 \cdot 8$ & No & Winter & 160 & 46 & Sporadic HUS & Reduced \\
\hline 10 & 9.0 & No & Winter & 60 & 9 & Sporadic HUS & Reduced \\
\hline 11 & $9 \cdot 2$ & No & Spring & 2310 & 258 & Sporadic HUS & Reduced \\
\hline 12 & $13 \cdot 2$ & Yes & Autumn & 1940 & 149 & Sporadic HUS & Reduced \\
\hline 13 & $13 \cdot 5$ & No & Autumn & 340 & 8 & Sporadic HUS & Normal \\
\hline
\end{tabular}

Conversion: SI to traditional units-creatinine $1 \mu \mathrm{mol} / \mathrm{l} \approx 0.01 \mathrm{mg} / 100 \mathrm{ml}$.

children aged 5 years to 13.5 years in whom HUS developed insidiously unassociated with a summer epidemic were considered to have sporadic HUS. Two of this latter group had recurrent disease (cases 8 and 13). One 9 year old girl (case 10) had a microangiopathic haemolytic anaemia and thrombocytopenia, but normal renal function.

To assess the specificity of $\mathrm{PGI}_{2}$ abnormalities for HUS another group of 9 children aged 10 months to 13 years with various other renal diseases was studied. This group comprised 1 child with acute nephritis, 2 with chronic renal failure due to HenochSchonlein nephritis, 1 with steroid sensitive nephrotic syndrome, 1 with focal glomerulosclerosis, 2 with shock and renal failure, and 2 with hypertension due to renovascular disease.

\section{Methods}

Nine volumes of venous blood from patients and normal adult controls were each collected into 1 volume of $3.8 \%$ trisodium citrate. Platelet poor plasma (PPP) was obtained within 20 minutes of collection by centrifugation at $1300 \mathrm{~g}$ for 10 minutes at room temperature and was either tested immediately or stored at $-20^{\circ} \mathrm{C}$ until required.

Platelet rich plasma (PRP) for aggregation studies was prepared from normal adults by centrifugation of citrated blood at $300 \mathrm{~g}$ for 15 minutes at room temperature. The final platelet count was adjusted to $250-300 \times 10^{9} / 1\left(250000-300000 / \mathrm{mm}^{3}\right)$ by dilution with autologous PPP.

Ability to support $\mathbf{P G I}_{2}$ production. The ability of plasma to support $\mathrm{PGI}_{2}$ production by rat aortic rings was assessed by a modification of the method of Moncada et al. ${ }^{14}{ }^{-} \mathrm{PGI}_{2}$ like activity produced being measured by the inhibitory effect on aggre- gation of normal human platelets challenged with $2.5 \mathrm{mmol} / 1$ adenosine diphosphate (ADP).

Aortic rings (1-2 mm width) were obtained from freshly sacrificed male Wistar rats. After rinsing 3 times in barbitone buffered saline (BBS), $\mathrm{pH} 7 \cdot 4$, the rings were kept in $\mathrm{BBS}$ at $0^{\circ} \mathrm{C}$ for up to 3 hours until used. In each experiment an aortic ring was incubated at $37^{\circ} \mathrm{C}$ for 5 minutes with $300 \mu \mathrm{PPP}$ from either a patient or a control subject, or with $300 \mu \mathrm{l}$ BBS. A $100 \mu \mathrm{l}$ aliquot was then removed and added to $300 \mu \mathrm{l}$ normal PRP. The mixture was stirred for 1 minute in a Payton aggregometer and ADP was then added to a final concentration of $2.5 \mu \mathrm{mol} / 1$. In the presence of PPP which had not been incubated with an aortic ring, full aggregation occurred. Inhibition of aggregation was taken to represent the effect of $\mathbf{P G I}_{2}$ like material secreted from the aortic ring into the plasma tested. Each ring that had been incubated with test plasma was then rinsed in BBS for $\mathbf{3 0}$ seconds and reincubated in control plasma (and vice versa) for 5 minutes before retesting, so that each ring acted as its own control.

Detection of an inhibitor. To distinguish between inhibition of $\mathrm{PGI}_{2}$ production and lack of $\mathrm{PGI}_{2}$ stimulating factors a second set of cross over experiments were performed in which the production of $\mathrm{PGI}_{2}$ like activity by each aortic ring incubated in test plasma was compared with that produced by the same ring following incubation in BBS. Diminished $\mathrm{PGI}_{2}$ production after incubation of aortic rings in HUS plasma compared with $\mathrm{PGI}_{2}$ production in BBS was taken to indicate the presence of an inhibitor.

Degradation of $\mathrm{PGI}_{2}$ in HUS plasma. $\mathrm{PGI}_{2}$ was added to $500 \mu \mathrm{l}$ PPP from patients in cases 8,11 , and 12 or normal control PPP in final concentrations of 20-100 ng/l. After incubation for 5 minutes at 
$37^{\circ} \mathrm{C}, 100 \mu \mathrm{l}$ PPP was removed and added to $400 \mu \mathrm{l}$ control PRP. After stirring for 1 minute in the aggregometer, ADP was added to $2.5 \mu \mathrm{mol} / 1$ final concentration and aggregation recorded.

Fractionation studies. Crude fractionation of plasma from the patient in case 8 into albumin rich and globulin rich fractions was performed using $45 \%$ saturated ammonium sulphate. Purification of the IgG fraction was achieved by ion exchange chromatography using DEAE cellulose. Fractionation of plasma proteins into low, medium, and high molecular weight ranges was performed by chromatography on a Sephadex G200 column. All fractions were concentrated to their original plasma concentrations by positive pressure filtration. All samples were prepared in phosphate buffered saline (PBS) pH $7 \cdot 2$ without azide.

Lipid extraction. Neutral lipids were extracted from $2 \mathrm{ml}$ plasma from the patients in cases 8 and 11 by mixing with $8 \mathrm{ml}$ hexane for 15 minutes,'centrifuging at $1300 \mathrm{~g}$ at $4^{\circ} \mathrm{C}$, removing the hexane layer, and evaporating the solvent under nitrogen. The residue was redissolved in $1 \mathrm{ml}$ BBS.

Polar lipids were extracted into a 1:1 mixture of cyclohexane and ethyl acetate after acidification to pH 3-4. After mixing for 15 minutes the solvent was separated and evaporated under nitrogen. The residue was again dissolved in BBS and tested for ability to support or inhibit $\mathrm{PGI}_{2}$ production.

\section{Results}

Plasma from 5 of 6 children with sporadic HUS showed decreased ability to support $\mathrm{PGI}_{2}$ production by aortic rings (Fig. 1, Table). No abnormality of $\mathrm{PGI}_{2}$ production was detected on the incubation of aortic rings with plasma from any of the 7 children with epidemic HUS or of the 9 non HUS patients when compared with the normal adult controls.

Less $\mathrm{PGI}_{2}$ production occurred when aortic rings were incubated in plasma from 3 of 4 sporadic HUS patients tested (no comparison with BBS made in the

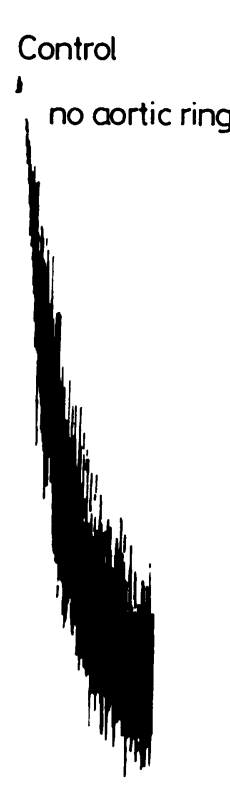

a

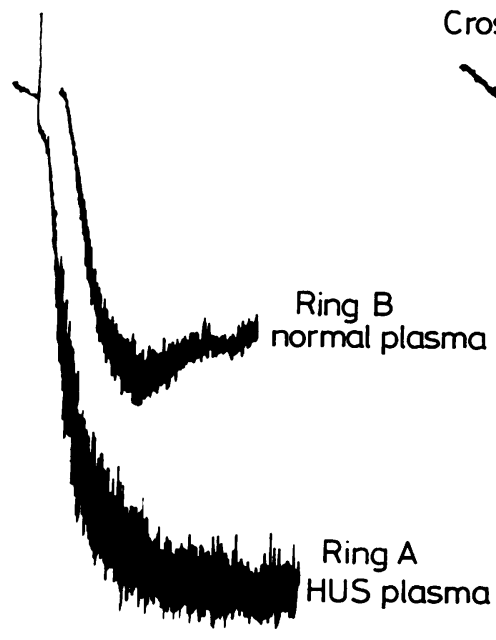

$\mathrm{b}$

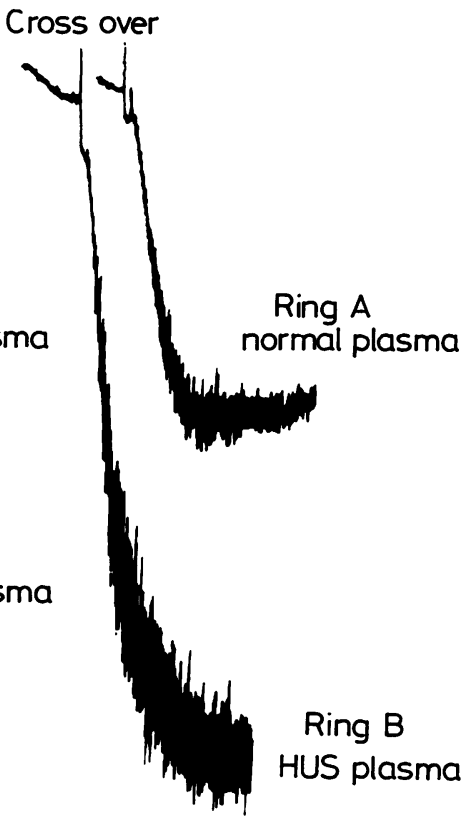

C

Fig. 1 Diminished $P G I_{2}$ production on incubation of plasma from the patient in case 8 with aortic rings

(a) Without prior incubation with an aortic ring, patient's plasma has no inhibitory effect on platelet aggregation induced by adenosine diphosphate.

(b) After incubation with aortic rings, patients' plasma (ring A) shows much less inhibition of aggregation than control plasma (ring B).

(c) Ring $A$ now incubated with normal plasma and ring $B$ with patient's plasma; only the normal plasma is inhibitory. HUS $=$ haemolytic uraemic syndrome. 

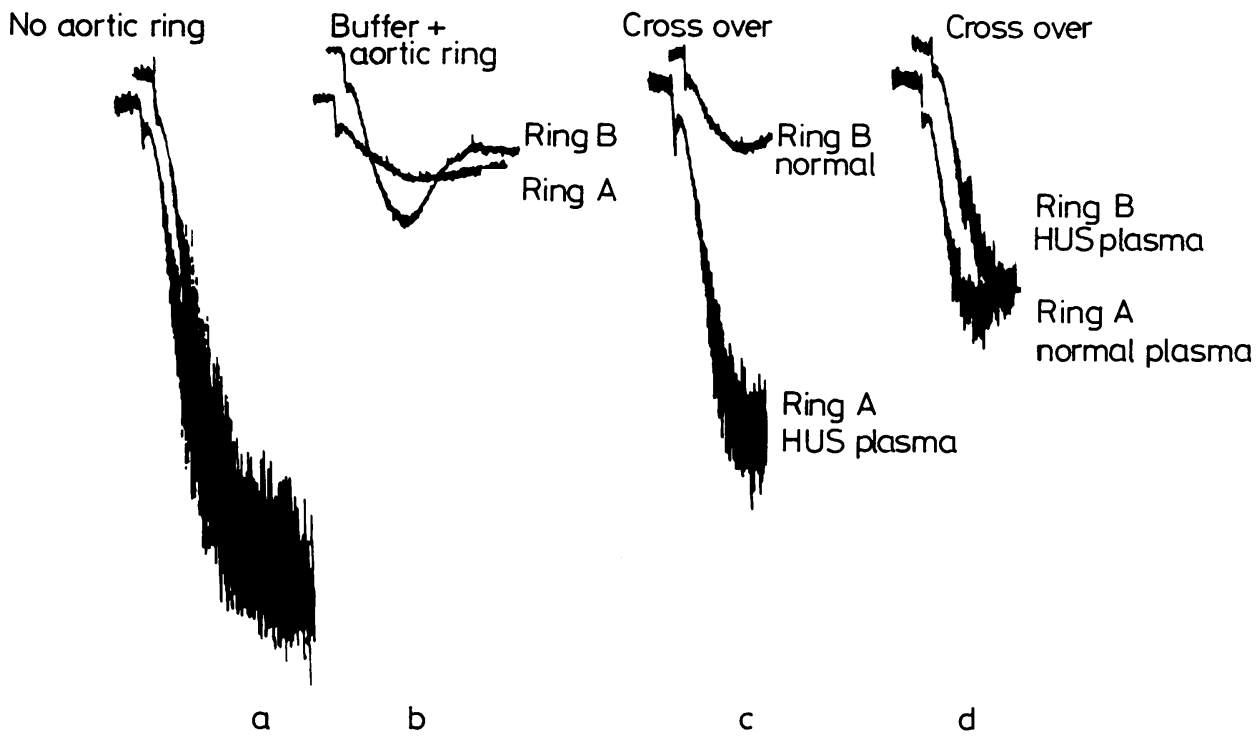

Fig. 2 Inhibition of $P I_{2}$ production by aortic rings incubated with HUS plasma from the patient in case 12.

(a) Without prior incubation with an aortic ring, patient's plasma and plasma from a normal control has no inhibitory effect on platelet aggregation induced by adenosine diphosphate.

(b) After incubation with aortic rings $A$ and $B$, barbitone buffered saline (BBS) inhibits platelet aggregation.

(c) Ring $A$ now incubated with patient's plasma ring $B$ with control plasma. The normal plasma is more inhibitory than $B B S$ after incubation with ring $B$, patient's plasma is much less inhibitory.

(d) Reincubation of ring $A$ with control plasma produces more, and incubation of ring $B$ with patient's plasma, less inhibitory activity than the previous reverse incubations.

HUS $=$ haemolytic uraemic syndrome.

patients in cases 9 and 11) than when the same ring was incubated in BBS (Fig. 2). This suggests inhibition of $\mathrm{PGI}_{2}$ production by HUS plasma rather than lack of stimulation. No such inhibitory activity was detected using plasma from the patient in case 13, from any of the epidemic HUS patients, the non HUS patients, or the controls.

The rate at which added $\mathrm{PGI}_{2}$ lost its activity on incubation with plasma from patients in cases 8,11, and 12 , determined by inhibition of platelet aggregation, was no different from that in the presence of normal plasma. This indicates that the effect of these patients' plasma was on the production rather than the degradation of $\mathrm{PGI}_{2}$.

Stability and fractionation studies. Fresh plasma from the patient in case 8 or her plasma stored at $-20^{\circ}$ for up to 3 months inhibited $\mathrm{PGI}_{2}$ production, but plasma stored at $4^{\circ} \mathrm{C}$ for 72 hours or dialysed for 72 hours showed neither inhibitory activity nor a failure to stimulate $\mathrm{PGI}_{2}$ production compared with normal plasma. Heating to $56^{\circ} \mathrm{C}$ for 30 minutes resulted in only a partial loss of the inhibitory activity. No inhibitory activity was detected in the protein fractions, which supported normal $\mathbf{P G I}_{2}$ production, but as these were prepared at $4^{\circ} \mathrm{C}$ or dialysed, inhibitory activity may have been lost.

The cyclohexane/ethyl acetate extracted fraction of plasma from patients in cases 8 and 9 inhibited $\mathrm{PGI}_{2}$ production whereas the same fraction of normal plasma had no inhibitory activity (Fig. 3). No inhibitory activity was detected in the hexane extracted fraction of either HUS or normal plasma.

\section{Discussion}

We have shown that plasma from certain patients with childhood HUS fails to support $\mathrm{PGI}_{2}$ production by aortic rings. This parallels previous reports on adult patients with HUS. The abnormality was found only in patients with sporadic HUS, however, and no abnormality of $\mathrm{PGI}_{2}$ production was detected in patients with the commoner epidemic form of HUS or in the other renal diseases studied.

Our findings suggest therefore that there may be different pathogenetic mechanisms in the epidemic 


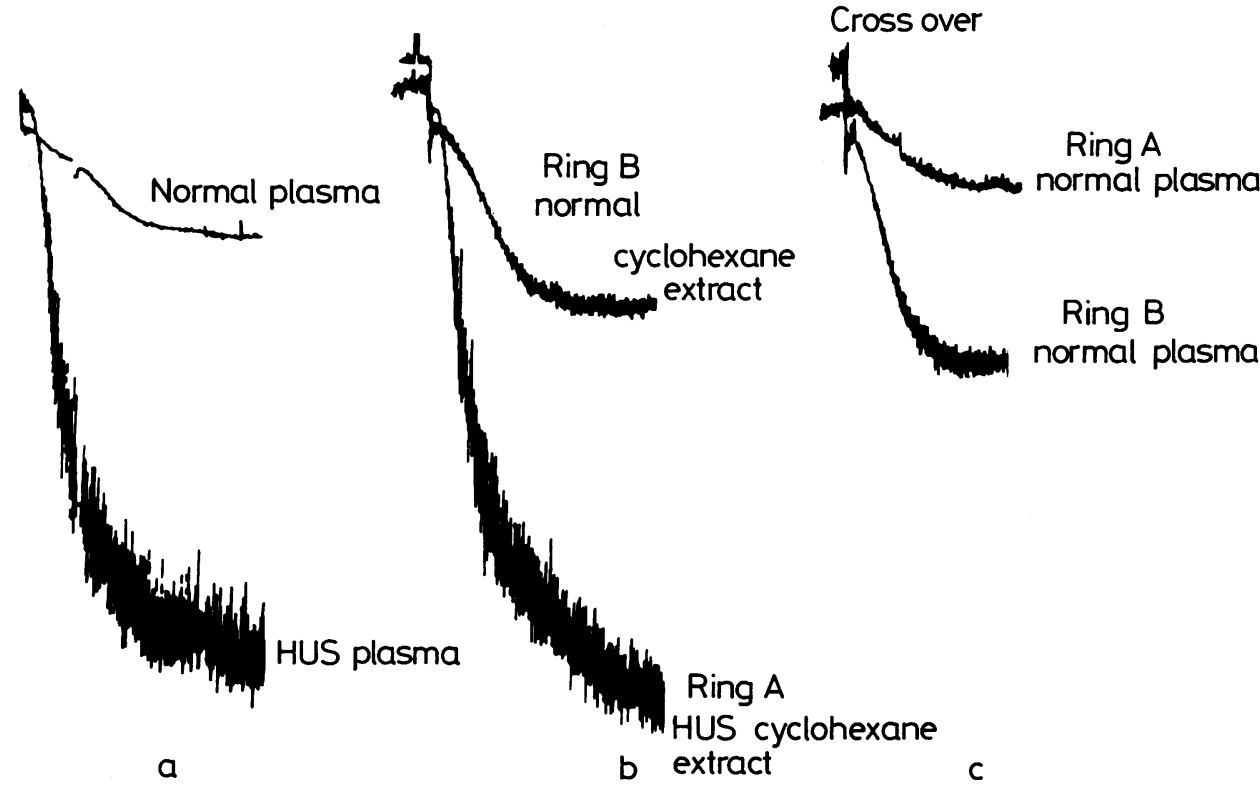

Fig. 3 Inhibition of $\mathrm{PGI}_{2}$ production by the cyclohexane/ethyl acetate extracted fraction of plasma from patient in case 8 .

(a) After incubation with aortic rings, control plasma inhibits platelet aggregation but patient's plasma does not.

(b) After incubation with aortic rings, the cyclohexane ethyl acetate extract of control plasma (ring B) inhibits platelet aggregation but the extract from patient's plasma (ring $A$ ) does not.

(c) After rinsing and incubation with control plasma, ring $A$ produces more inhibitory activity than ring $B$.

HUS = haemolytic uraemic syndrome.

and sporadic HUS subgroups and add weight to the epidemiological, ${ }^{11}$ genetic, ${ }^{15}$ and histological ${ }^{16}$ evidence suggesting that HUS comprises at least 2 distinct diseases that differ not only in their epidemiology, clinical features, and prognosis but also in their underlying pathogenesis. Therapeutic trials and laboratory investigations of HUS need to recognise the heterogeneity of patients with the syndrome.

Previous investigators have ascribed the abnormality of $\mathbf{P G I}_{2}$ production on incubation of endothelial tissue with HUS plasma to either lack of a stimulating factor or excessively rapid degradation of $\mathrm{PGI}_{2}$. Our observation, however, that less $\mathrm{PGI}_{2}$ production occurred on incubation of aortic rings in some samples of HUS plasma than occurred in BBS alone suggests the presence of an inhibitor of $\mathrm{PGI}_{2}$ production in them. Furthermore, prolonged storage or dialysis at $4^{\circ} \mathrm{C}$ resulted in loss of the inhibitory activity from HUS plasma which then showed normal ability to support PGI $_{2}$ production. There was no evidence of execssive degradation of added $\mathrm{PGI}_{2}$ in our patients' plasma. Although the cause of the abnormality of $\mathrm{PGI}_{2}$ production in our patients thus differs from that proposed by other workers, it is possible that more than 1 mechanism may induce $\mathrm{PGI}_{2}$ deficiency in different groups of patients.

Our preliminary studies to characterise the inhibitor of $\mathrm{PGI}_{2}$ production indicate that it is a polar lipid, extractable into a mixture of cyclohexane and ethyl acetate. It is of interest that 1 of the earliest known inhibitors of $\mathrm{PGI}_{2}$ synthesis was 15 hydroxyperoxyarachidonic acid ${ }^{17}$ and other lipid peroxides are also known to inhibit $\mathrm{PGI}_{2}$ synthesis. ${ }^{18}$ Several authors have reported reduced concentrations of antioxidants such as vitamin $E,{ }^{19}$ superoxide dismutase, ${ }^{20}$ and transferrin ${ }^{21}$ in patients with HUS. It is therefore possible that the inhibitor of $\mathrm{PGI}_{2}$ production in the sporadic HUS patients is a lipid peroxide (possibly an arachidonic acid metabolite) produced after an oxidizing insult or resulting from lack of antioxidants in HUS plasma. Further studies are required to define the nature of the inhibitor more precisely and to establish whether its presence is related to the reported defects in antioxidant potential in HUS plasma.

We thank Wellcome Laboratories for the gift of PGI . ML was supported by a grant from both the Kidney Research Aid Fund and the National Kidney Research Fund. 


\section{References}

1 Remuzzi G, Marchesi D, Mecca G, et al. Haemolyticuraemic syndrome: deficiency of plasma factor(s) regulating prostacyclin activity ? Lancet 1978 ;ii:871-2.

2 Webster J, Rees AJ, Lewis PJ, Hensby CN. Prostacyclin deficiency in haemolytic uraemic syndrome. $\mathrm{Br}$ Med $J$ 1980;281:271.

3 Machin SJ, Defreyn G, Chamone DAF, Vermylen J. Plasma 6-keto-PGF $\propto$ levels after plasma exchange in thrombotic thrombocytopenic purpura (letter). Lancet $1980 ; \mathbf{i}: 661$.

4 Chen YC, Hall ER, McLeod B, Wu KK. Accelerated prostacyclin degradation in thrombotic thrombocytopenic purpura. Lancet 1981 ;ii:267-9.

5 MacIntyre DE, Pearson JD, Gordon JL. Localisation and stimulation of prostacyclin production in vascular cells. Nature 1978;271:549-51.

6 Byrnes JJ, Khurana M. Treatment of thrombotic thrombocytopenic purpura with plasma. N Engl J Med 1977; 297:1386-9.

7 Anonymous. Plasma exchange in thrombotic thrombocytopenic purpura (Editorial). Lancet 1979;i:1065-6.

8 Pini M, Manotti C, Quintavalla R, Dettori AG. Normal prostacyclin-like activity in thrombotic thrombocytopenic purpura (letter). Thromb Haemost $1981 ; 46: 571$.

9 Fong JSC, de Chadarevian JP, Kaplan BS. Hemolyticuremic syndrome: current concepts and management. Pediatr Clin North Am 1982;29:835-56.

10 Anonymous. Platelets, endothelium and renal disease (Editorial). Lancet 1979 ;ii:890-2.

11 Trompeter RS, Schwartz R, Chantler C, et al. Haemolyticuraemic syndrome: an analysis of prognostic features. Arch Dis Child 1983;58:101-5.

12 Ridolfi RL, Bell WR. Thrombotic thrombocytopenic purpura; report of 25 cases and review of the literature. Medicine (Baltimore) 1981 ;60:413-28.
13 Gianantonio CA, Vitacco M, Mendilaharzu F, Gallo GE, Sojo ET. The hemolytic-uremic syndrome. Nephron 1973;11:174-92.

14 Moncada S, Higgs EA, Vane JR. Human arterial and $\overrightarrow{\vec{F}}$ venous tissues generate prostacyclin (prostaglandin X), a potent inhibitor of platelet aggregation. Lancet 1977;i: 18-21.

15 Kaplan BS, Chesney RW, Drummond KN. Hemolytic uremic syndrome in families. $N$ Engl $J$ Med 1975;292: 1090-3.

16 Habib R, Levy M, Gagnadoux MF, Broyer M. Prognosis of the hemolytic-uremic syndrome in children. $A d v$ Nephrol 1982;11:99-128.

17 Moncada S, Vane JR. Prostacyclin, platelet aggregation and thrombosis. In: De Gaetano G, Garattini S, eds. Platelets: A multi-disciplinary approach. New York: Raven Press, 1978:239-58.

18 Ham EA, Egan RW, Soderman DD, Gale PH, Kuehl FA, 응 Jr. Peroxidase-dependent deactivation of prostacyclin synthetase. J Biol Chem 1979;254:2191-94.

19 O'Regan S, Chesney RW, Kaplan BS, Drummond KN. ?o Red cell membrane phospholipid abnormalities in the $\checkmark$ hemolytic-uremic syndrome. Clin Nephrol 1980;15:14-7. C

20 Kobayashi Y, Okahata S, Tanabe K, Tanaka Y, Veda K, Usui T. Erythrocyte syperoxide dismutase activity in hemolytic-uremic syndrome. Hiroshima J Med Sci 1978;27:181-3.

21 Donati MN, Misiani R, Marchesi D, et al. Hemolyticuremic syndrome, prostaglandins and plasma factors. In: Remuzzi G, Mecca G, De Gaetano G, eds. Hemostasis, prostaglandins and renal diseases. New York: Raven Press, 1980:273-90.

Correspondence to: Dr M Levin, Department of Nephrolog: Institute of Child Health, London, WC1N 1EH.

Received 25 May 1983 